\title{
Nurses, Covid-19 pandemic and Saudi Arabia
}

\author{
Ahmad Aboshaiqah ${ }^{1,} *$, Mohammed G. Alghamdi ${ }^{2}$ \\ ${ }^{1}$ College of Nursing, King Saud University, Riyadh, Saudi Arab \\ ${ }^{2}$ Director General Nursing Affairs General Department, Ministry of Health, Riyadh, Saudi \\ Arabia
}

\section{*Correspondence}

Dr. Ahmad Aboshaiqah, RN, PhD.

College Of Nursing

King Saud University, Riyadh, Saudi Arabia

Email: aaboshaiqah@ksu.edu.sa;

ashaiqahksu@gmail.com 


\section{INTRODUCTION}

In a timeline that approaches the present day, the World Health Organization (WHO) Country Office in China first recorded an outbreak with such an unparalleled nature of unexplained lower respiratory infections in Wuhan, the largest metropolitan city in China's Hubei province, on 31 December 2019. The Chinese Center for Disease Control and Prevention (CDC) and local CDCs organized an intensive outbreak investigation program to fight against this new infection. The etiology of this illness is now attributed to a novel virus belonging to the coronavirus $(\mathrm{CoV})$ family (Cascella et al., 2020). The current outbreak is reported to be caused by severe acute respiratory syndrome corona virus 2 (SARS-CoV-2), which is from the same family of viruses accountable for Middle East respiratory syndrome (MERS) and severe acute respiratory syndrome (SARS) (Bchetnia et al., 2020). On February 11, 2020, the WHO announced that the disease caused by this new CoV was named "COVID-19," which is the acronym of "coronavirus disease 2019" (WHO). This new virus is very contagious and is spreading very quickly around the globe. With such huge public health impact all over the world, WHO declared COVID19 as first pandemic of the 21 st century [www.who.int]. As of December 22, the number of COVID-19 cases has reached more than 70 million with more than 1.7 million deaths (https://www.worldometers.info/coronaviru) . In Saudi Arabia, the outbreak was taken very seriously from the very beginning even before the first case was reported on March 2, 2020. The Saudi government has undertaken rapid and swift actions, such as suspending Omrah and holding international flights. Such affirmative actions enhanced citizens and residents' confidence in the government officials' ability to fight the pandemic. However, there are more than 361,178 
reported cases of Covid-19 with 6131 deaths

as of December 22, 2020

[www.worldometer.com/corona].

As nurses are the largest component of the health care workforce in Saudi Arabia, it is important that they are trained and have the skills and resources to adapt, whether on the frontline of a crisis or in a clinical environment. Nurses play a key function in fulfilling the needs of medical treatment by being the most important part of the health care delivery system (Pope et al., 19995; AlYami and Watson, 2012). Through this pandemic, the Nurses in Saudi Arabia proved to be the defense fighters who took up the challenge with their hearts and souls and with great resolve. Nurses in Saudi Arabia are training around the clock, operating on the brink, putting their lives on a pause during the early crises of the latest pandemic. In the early days of the latest epidemic, the whole healthcare sector has experienced alarming rates of overwork in different health care facilities, such as intensive care units and emergency departments. Nurses here are often seen operating without sufficient rest and rehabilitation, with little regard for their mental health and well-being, without help and assistance. Yet they freely and gladly embrace these obligations, responsibility, and obstacles throughout the Kingdom. Nurses are a significant link between the patient and other health care providers because they are with their patients all the time while coping with the latest Covid-19 crises, and are able to detect subtle changes in their patients by observation and analytical thinking that may suggest that they are progressing or worsening (Fernandez et al., 2020).

Furthermore, the healthcare system in Saudi Arabia is categorized as a national system, which provides health services through governmental sectors and agencies for a total population of 32.5 million. Although Saudi Arabia has a high gross 
domestic product for its population, it has a shortage of registered nurses at only 57 per 10,000 population compared to the US at 98/ 10,000 or Japan at $112 / 10,000$ population. Therefore, health care sectors in Saudi Arabia need of additional $54 \mathrm{~K}$ nurses by the end of 2022 to achieve the target of 70 nurses per 10,000 population as an international benchmark (Saudi MOH, 2020; Figure 1).

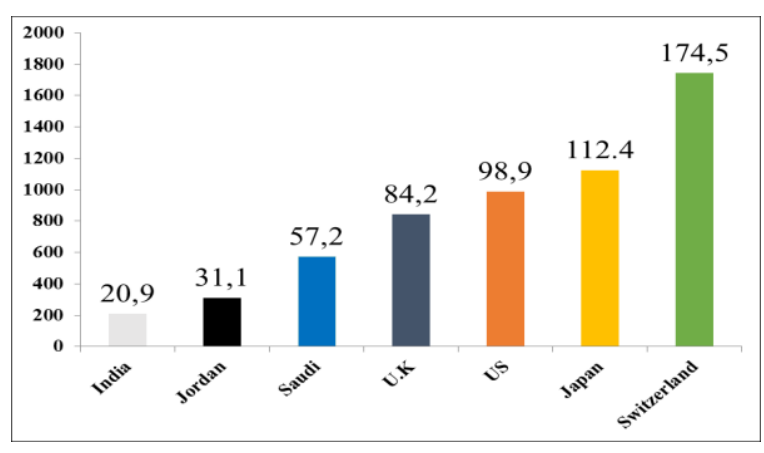

Figure 1. The total number of nursing and midwifery personnel per 10000 population.

During the pandemic, nursing shortage become a major concern as the number of confirmed COVID-19 cases accelerates dramatically. Additionally, the infection among nurses was noticed in the beginning of the pandemic due to several factors. The workforce shortage; lack of competency-based training regarding COVID-19 case; lack of guidelines and protocols; workload, stress, and burnout were considered the most influencing factors. Therefore, the Saudi Ministry of Health adopted several short-term and long-term strategies to cover the nursing shortage such as implementing the nursing rapid-response team, building a nursing surge capacity plan, and adopting the national nursing retention strategy. Furthermore, the high number of infection among nurses has led the Saudi Ministry of Health to take the cross-infection indicator as a crucial outcome to reflect nurses' adherence to the clinical guidelines and protocols, patient safety measures and standard precautions, infection control policies including hand hygiene compliance. The proactive roles that were taken by the Ministry of Health have resulted in a very notable decrease in the confirmed infected nurses with coronavirus cases. Nurses have played a critical role in providing quality 
care, infection prevention, containment, and infection control during COVID-19 pandemic (Alghamdi, 2020). MOH across the Kingdom has made relentless efforts to combat this Covid-19 pandemic by offering unique training to all medical fraternity who are like frontline warriors. Saudi Arabia 's nurses are constantly updated with new Covid-19 pandemic policies and procedures, they are provided with education, training, and their morals are boosted to fight back the pandemic. Across all health care facilities throughout the Kingdom, nurses have been trained on basic infection management techniques to deal with suspected or confirmed cases of Covid-19. In the current outbreak, $\mathrm{MOH}$ has established a nursing leadership and control unit to oversee health facilities and nursing sections with the purpose of assigning nursing personnel to health facilities in emergencies, identifying possible risks and ensuring rapid response. It also monitors the nursing staff around the clock, as well as the rapid response teams in hospitals and healthcare sectors and in quarantine establishments.

During this pandemic nurses in Saudi Arabia provide health education, screening services, and support for the general public and high-risk individuals. By practicing hand hygiene and using personal protective equipment they help in prevention of infection. Nurses screen suspected cases by writing their case and travel histories. They prevent the nosocomial infections by educating and training patients, families and other health workers to contain the spread of Covid-19. Nurses are planning and implementing appropriate preparations and precautions in covid-19 designated hospitals across the Kingdom regarding care for covid19 patients who required long-term care as these patients were more vulnerable to other infections. Patients with immune deficiencies or underlying diseases, particularly chronic obstructive pulmonary diseases, chronic 
diseases, hematological disorders and other serious diseases, have a higher risk of infection with Covid-19 than other patients without underlying disease (Ejaz et al., 2020). Therefore, educating this vulnerable class of patients on self-protection strategies is a critical function of nurses (American Society of Clinical Oncology, 2020; CDC, 2020). While most of the covid-19 patients appear with mild symptoms and isolate themselves at home and recover with a week or two, there are some group of people who experience serious Covid-19 symptoms and require hospitalization for sure. Nurses in Saudi Arabia are taking a good care of these kind of patients in emergency units, general Covid-19 units and ICUs. All health care providers are using adequate PPEs to prevent themselves from catching infection. In intensive care, nursing staffs provide direct life-sustaining care to help patients recover from COVID-19-induced pneumonia as well as provide psychological support to patients in states of panic or distress (Al Thobaity et al., 2020).

As the world and the Kingdom have shown, nurses tend to be the ones to share the burden of healthcare and are grateful that their contribution is being recognized more than ever. Globally, the way the world treats nurses and health-care practitioners is also changing. At a time of pandemic, nurses are very conscious of the complexities of providing high-quality care and have demonstrated how they can boost profitability with consistent high quality. The Covid-19 pandemic has brought nurses to the forefront of people's minds, and daily capture of media attention and public gratitude to health care professionals. As not everyone understands what is happening in a health care facility, the Covid-19 pandemic has emphasized the importance of the nurses' contribution to prevention and promotion of health care. In these difficult times, the reputation of the nursing community and 
culture, and the overall morale of nursing, is gaining considerable attention and appreciation.

\section{Funding. None}

Conflict of Interest. None

\section{References:}

1. Cascella M, Rajnik M, Cuomo A, et al. Features, Evaluation, and Treatment of Coronavirus. [Updated 2020 Oct 4]. In: StatPearls [Internet]. Treasure Island (FL): StatPearls Publishing; 2020 Jan-. Available from: https://www.ncbi.nlm.nih.gov/books/NB K554776/

2. Bchetnia M, Girard C, Duchaine C, Laprise C. The outbreak of the novel severe acute respiratory syndrome coronavirus 2 (SARS-CoV-2): A review of the current global status. J Infect Public Health. 2020 Nov;13(11):16011610. doi: 10.1016/j.jiph.2020.07.011. Epub 2020 Aug 4. PMID: 32778421; PMCID: PMC7402212.

3. World Health organization (WHO). https://covid19.who.int/

4. Worldometers 2020. https://www.worldometers.info/coronavi rus/

5. AlYami MS, Watson R. An overview of nursing in Saudi Arabia. J Health Spec [serial online] 2014 [cited 2020 Dec 10];2:10-2.

6. Pope AM, Snyder MA, Mood LH, editors. Nursing Health, \& Environment: Institute of Medicine (US) Committee on
Enhancing Environmental Health Content in Nursing Practice; Strengthening the Relationship to Improve the Public's Health. Washington (DC): National Academies Press (US); 1995. 3, Nursing Practice

7. Fernandez R, Lord H, Halcomb E, Moxham L, Middleton R, Alananzeh I, Ellwood L. Implications for COVID-19: A systematic review of nurses' experiences of working in acute care hospital settings during a respiratory pandemic. Int J Nurs Stud. 2020 Nov;111:103637. doi: 10.1016/j.ijnurstu.2020.103637. Epub 2020 May 8. PMID: 32919358; PMCID: PMC7206441.

8. Ministry of Health $(\mathrm{MOH})$, Kingdom of Saudi Arabia. ttps://www.moh.gov.sa/en/HealthAware ness/EducationalContent/PublicHealth/P ages/corona.aspx

9. Alghamdi, M. G., (2020). Challenges Facing Nurses in Saudi Arabia during the COVID-19 Pandemic. Saudi Patient Safety Center Newsletter. 2(2), 9-11

10. Ejaz H, Alsrhani A, Zafar A, Javed H, Junaid K, Abdalla AE, Abosalif KOA, Ahmed Z, Younas S. COVID-19 and comorbidities: Deleterious impact on infected patients. J Infect Public Health. 2020 Dec;13(12):1833-1839. doi: 10.1016/j.jiph.2020.07.014. Epub 2020 Aug 4. PMID: 32788073; PMCID: PMC7402107.

11. American Society of Clinical Oncology. https://www .asco.org/asco-coronavirusinformation/care-individuals-cancerduring-covid-19 
12. Centers for Disease Control and Prevention (CDC).

https://www.cdc.gov/media/dpk/diseases -and-

conditions/coronavirus/coronavirus-

2020.html

13. Al Thobaity A, Alshammari F. Nurses on the Frontline against the COVID-19 Pandemic: An Integrative Review. Dubai Medical Journal. 2020 Aug 26:1-6. doi: 10.1159/000509361. PMCID:

PMC7490501. 